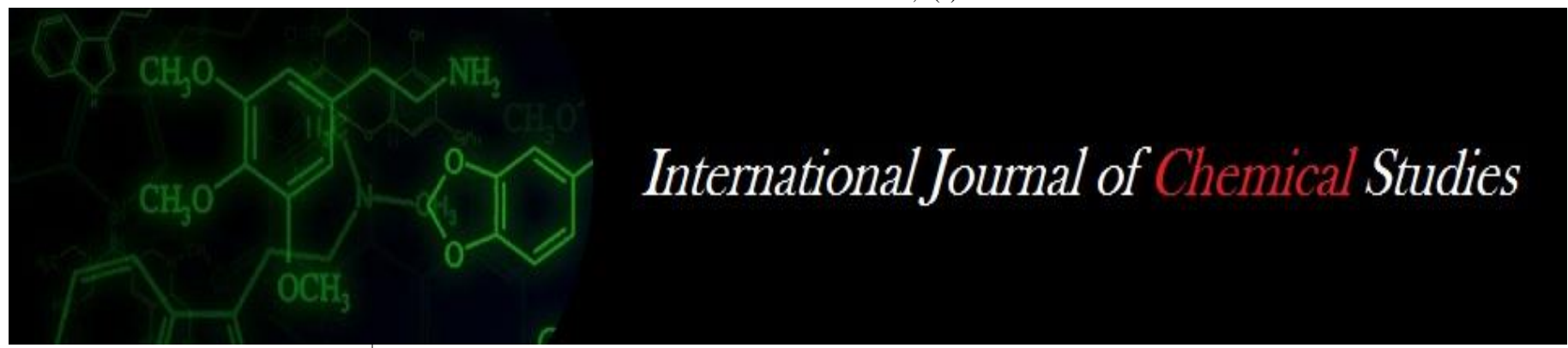

P-ISSN: 2349-8528

E-ISSN: 2321-4902

www.chemijournal.com

IJCS 2020; 8(2): 2742-2746

(C) 2020 IJCS

Received: 19-01-2020

Accepted: 21-02-2020

\section{Y Satish}

Regional Agricultural Research Station, Acharya N.G. Ranga Agricultural University, Lam, Guntur, Andhra Pradesh, India

\section{Sudha Rani}

Regional Agricultural Research

Station, Acharya N.G. Ranga

Agricultural University, Lam,

Guntur, Andhra Pradesh, India

\section{Rani Chapara}

Regional Agricultural Research Station, Acharya N.G. Ranga Agricultural University, Lam, Guntur, Andhra Pradesh, India
Corresponding Author:

Y Satish

Regional Agricultural Research Station, Acharya N.G. Ranga Agricultural University, Lam, Guntur, Andhra Pradesh, India

\section{Correlation and path coefficient analysis for yield and yield component traits in upland cotton (Gossypium hirsutum L.)}

\author{
Y Satish, M Sudha Rani and Rani Chapara
}

DOI: https://doi.org/10.22271/chemi.2020.v8.i2ap.9166

\begin{abstract}
This experiment was carried out with 40 genotypes of cotton in randomized complete block design (RCBD) with three replications at Regional Agricultural Research Station, Lam, Guntur during kharif, 2019-20. Each plot consisted of one row of $6 \mathrm{~m}$ length and observations were recorded on five randomly selected plants from each genotype per replication for characters viz., plant height $(\mathrm{cm})$, number of monopodia plant per plant, number of sympodia plan per plant, number of bolls plant per plant, boll weight $(\mathrm{g})$, seed index $(\mathrm{g})$, lint index $(\mathrm{g})$ and seed cotton yield plant per plant $(\mathrm{g})$. The characters viz., Days to $50 \%$ flowering, ginning out turn $(\%), 2.5 \%$ span length $(\mathrm{mm})$, micronaire $\left(10^{-6} \mathrm{~g} / \mathrm{inch}\right)$, bundle strength $(\mathrm{g} / \mathrm{tex})$ and uniformity ratio were recorded on plot basis. Correlation and path coefficient analysis were worked out for 14 characters among 40 genotypes of upland cotton. Correlation studies revealed that plant height, number of sympodia per plant, number of bolls per plant and boll weight recorded significant positive association with seed cotton yield per plant. Further partitioning of correlation coefficients into direct and indirect effects showed that characters, plant height, number of bolls plant per plant, boll weight, ginning out turn (GOT) and micronaire value had direct positive effect on seed cotton yield per plant. Thus, correlation and path analysis clearly indicated that direct selection based on number of bolls per plant, boll weight and seed index may be helpful in developing high yielding varieties in upland cotton.
\end{abstract}

Keywords: Correlation, path coefficient analysis, cotton

\section{Introduction}

Cotton (Gossypium spp.) popularly known as "King of fibre" and "White Gold" is one of the most important commercial cash crops and plays a key role in economic, political and social affairs of the world. Cotton enjoys a pre-eminent status among all the cash crops in the country, being the principal material for flourishing textile industries. The predominant species cultivated in India is $G$. hirsutum which covers about $90 \%$ of the total area. India is maintaining the position of leading cotton growers in the world, China leading in terms of cotton production. Although cotton is cultivated in 77 countries; the five countries - China, India, United States, Brazil and Pakistan, produces $78 \%$ of the total world production from $72 \%$ of the world gross cotton area. China and Bangladesh are being the largest net importers of cotton (19\% each) of the total world import, followed by Vietnam (17\%), Indonesia (8\%) and Pakistan (7\%). The United States maintaining leading exporter of cotton (36\%) of the total world export, followed by Brazil (14\%), India (10\%) and Australia (9\%). And the productivity front Australia leading with yield of $1814 \mathrm{~kg} / \mathrm{ha}$, followed by China (1726) and Brazil (1636) and India way behind at $507 \mathrm{~kg}$ lint/ha (AICCIP Annual Report, 2018-19)

The ultimate objective of any breeder is to increase the yield and is normally a complex trait governed by polygenes. Hence, it is desirable for plant breeder to know the extent of relationship between yield and yield components which will facilitate in selecting desirable characteristics for yield improvement. Correlation coefficient analysis measures the magnitude of relationship between various plant characters and determines the component character on which selection can be based for improvement of seed cotton yield. Further, the true picture of correlation between seed cotton yield and other yield traits is reflected from direct and indirect effects in order to perceive the most influencing characters to be utilized as selection criteria in cotton breeding programme. 


\section{Material and Methods}

The present study was carried out with 40 genotypes of cotton in randomized complete block design (RCBD) with three replications at Regional Agricultural Research Station, Lam, Guntur during kharif, 2019-20. The inter-row and intra-row spacing adapted was $105 \mathrm{~cm}$ x $60 \mathrm{~cm}$. Each plot consisted of two rows of $6 \mathrm{~m}$ length. The row and plant spacings adapted were 105 and $60 \mathrm{~cm}$, respectively. Recommended cultural practices were carried out and the crop was grown under uniform field condition to minimize environmental variations to the maximum possible extent. Observations were recorded on five randomly selected plants from each genotype per replication for characters viz., plant height $(\mathrm{cm})$, number of monopodia plant per plant, number of sympodia plan per plant, number of bolls plant per plant, boll weight $(\mathrm{g})$, seed index $(\mathrm{g})$, lint index $(\mathrm{g})$ and seed cotton yield plant per plant (g). The characters viz., Days to $50 \%$ flowering, ginning out turn $(\%), 2.5 \%$ span length $(\mathrm{mm})$, micronaire $\left(10^{-6} \mathrm{~g} /\right.$ inch), bundle strength (g/tex) and uniformity ratio were recorded on plot basis. The fibre quality characters were analysed at Central Institute for Research on Cotton Technology Regional Unit, Lam, Guntur. The fiber quality traits were evaluated with HVI (High Instrument Volume. The analysis of variance was carried out following Panse and Sukhatme (1978) ${ }^{[15]}$. Correlation coefficients between different characters were worked out as per Al-Jibouri et al. (1958) ${ }^{[2]}$. Genotypic correlation coefficients were further partitioned into direct and indirect effects by path analysis as suggested by Dewey and $\mathrm{Lu}(1959)^{[8]}$.

\section{Results and Discussions}

The analysis of variance revealed highly significant differences among the genotypes for all the characters studied. The genotypic and phenotypic correlation coefficients and the genotypic and phenotypic path coefficients showing direct and indirect effects are presented in Table 1, 2 and 3 respectively. In general higher genotypic correlation coefficients than the phenotypic correlation coefficients were observed in the present study which is in conformity with the findings of Desalegn et al. (2009) ${ }^{[7]}$ who reported chief role of genetic effects. This indicated the strong inherent association between characters governed largely by genetic causes and is generally less subjected to environmental forces.

Computation of correlation between yield and yield attributing traits is of considerable importance in plant selection. The traits, number of bolls per plant, plant height, number of monopodia per plant, number of sympodia per plant and boll weight were found to posses significant positive association with seed cotton yield per plant both at genotypic level. While, plant height, number of sympodia per plant, number of bolls per plant expressed significant positive association at phenotypic level also. Therefore, selecting high yielding plants based on, number of bolls per plant, boll weight and number of sympodia per plant will be more useful. Similar results were reported by Hazem et al. (2005) [10], Desalegn et al. (2009) ${ }^{[7]}$ and Ahsan et al. (2014) ${ }^{[14]}$.

In textile industry point of view, ginning outturn is very important because it shows the recovery of lint from seed cotton. In the present study, the traits days to $50 \%$ flowering, lint index, GOT and uniformity ratio exhibited significant negative genotypic association with seed cotton yield. However, these results are in contradict with the earlier reports of Ramesh (2015) ${ }^{[20]}$, Vinodhana et al. (2013) ${ }^{[22]}$ who reported the seed index, lint index and ginning outturn had significant positive correlation with seed cotton yield. Whereas, seed index, micronaire value and bundle strength recorded non significant genotypic correlation with seed cotton yield per plant.

However, correlation of yield and its components alone are not adequate in any selection programme. The inter relationship among the individual character may ultimate influence the yield. In the present study, plant height recorded significant positive association with number of sympodia per plant, number of bolls per plant, boll weight, seed index, bundle strength at both genotypic and phenotypic levels indicating their true association. Similar results were also reported earlier by Alkuddsi et al. (2013) ${ }^{[3]}$, Rajamani et al. (2013) ${ }^{[19]}$ and Asha et al. (2015) ${ }^{[5]}$. Days to $50 \%$ flowering showed significant negative association with boll weight, seed index and lint index both at phenotypic and genotypic levels. This was in accordance with the research findings of An et al. (2008) ${ }^{[4]}$, Kumar et al. (2010) ${ }^{[14]}$ and Sirisha et al. (2016) ${ }^{[21] .}$ The traits, number of monopodia per plant, number of sympodia per plant, number of bolls per plant, boll weight and seed index also showed significant positive association with seed cotton yield per plant at both phenotypic and genotypic levels indicating the usefulness of these traits in selection programmes. Similar results were reported by Kishore et al. (2011) [13] and Rajamani et al. (2013) [19]. Days to $50 \%$ flowering, ginning out turn and uniformity ratio showed nonsignificant negative association with seed cotton yield per plant at phenotypic level, whereas at genotypic level they show significant negative association. This was in accordance with Pujer et al. (2014) ${ }^{[18]}$ and Sirisha et al. (2016) ${ }^{[21]}$.

The correlation coefficient alone are insufficient to explain the relationship for effective manipulation of the characters, but path coefficient analysis furnishes a method for partitioning the correlation coefficient into direct and indirect effects and measures the relative importance of the causal factors in determining the seed cotton yield. The results of such analysis are discussed below.

In plant breeding, it is very difficult to have complete knowledge of all component traits of yield. The residual effect permits precise explanation about the pattern of interaction of other possible components of yield. In other words, residual effect measures the role of the possible independent variables which were not included in the study on the dependent variable. In the present study, the residual effect observed at phenotypic (0.539) and genotypic (0.125) explains that the characters chosen for path analysis were adequate and appropriate. Among the characters studied, the traits viz., plant height, number of bolls per plant, boll weight, ginning out turn, micronaire value and bundle strength showed direct positive effects besides expressing significant positive correlation with yield. Therefore, a direct selection of these traits is suggested for obtaining yield improvement. Similarly,

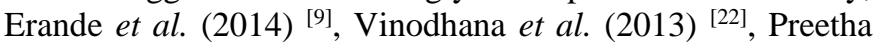
and Raveendran (2007) ${ }^{[16]}$, Ashok kumar and Ravikesavan (2010) ${ }^{[6]}$ observed positive and direct effect of one or other of above characters on seed cotton yield. However, significant negative direct effect was observed for number of monopodia per plant, number of sympodia per plant, lint index, $2.5 \%$ span length. Thus, these studies revealed that, the traits which had positive and direct effect on seed cotton yield should be given due to emphasis for making selection for high yielding genotypes.

In the present study, number of bolls per plant exhibited positive indirect effect on seed cotton yield via number of sympodia, whereas boll weight exhibited positive indirect 
effect on yield via seed index, $2.5 \%$ span length. Among the fibre quality traits, $2.5 \%$ span length exhibited positive indirect effect on yield via uniformity ratio and bundle strength via number of bolls per plant, boll weight, seed index, lint index and $2.5 \%$ span length. These results are in agreement with the findings of Kaushik et al. (2005) ${ }^{[12]}$ and Iqbal et al. (2006) ${ }^{[11]}$.

Table 1: Phenotypic (below diagonal) and genotypic (above diagonal) correlation coefficients for 14 characters among 40 genotypes of cotton (Gossypium hirsutum L.) during kharif, 2019-20

\begin{tabular}{|c|c|c|c|c|c|c|c|c|c|c|c|c|c|c|}
\hline Character & $\begin{array}{c}\text { Plant } \\
\text { height }\end{array}$ & $\begin{array}{c}\text { Days to } \\
50 \% \\
\text { flowering }\end{array}$ & $\begin{array}{c}\text { Number of } \\
\text { monopodia } \\
\text { per plant }\end{array}$ & $\begin{array}{c}\text { Number of } \\
\text { sympodia } \\
\text { per plant }\end{array}$ & $\begin{array}{c}\text { Number } \\
\text { of bolls } \\
\text { per plant }\end{array}$ & \begin{tabular}{|c} 
Boll \\
weight
\end{tabular} & $\begin{array}{l}\text { Seed } \\
\text { index }\end{array}$ & $\begin{array}{c}\text { Lint } \\
\text { index }\end{array}$ & $\begin{array}{l}\text { Ginning } \\
\text { outturn }\end{array}$ & $\begin{array}{c}2.5 \% \\
\text { span } \\
\text { length }\end{array}$ & $\underset{\text { ratio }}{\text { Uniformity }}$ & $\begin{array}{c}\text { Micronaire } \\
\text { value }\end{array}$ & $\begin{array}{c}\text { Bundle } \\
\text { strength }\end{array}$ & \begin{tabular}{|c|} 
Seed \\
cotton \\
yield per \\
plant \\
\end{tabular} \\
\hline \begin{tabular}{|c|} 
Plant height \\
$(\mathrm{cm})$
\end{tabular} & 1.0000 & $0.3264 * *$ & $2241^{*}$ & $0.8068 * *$ & 0.3555 & 0.0622 & $0.3529 * *$ & -0.1142 & $0.4272 * *$ & -0.0691 & $-0.3860 * *$ & 0.0214 & -0.1777 & $0.3284 * *$ \\
\hline \begin{tabular}{|c|} 
Days to \\
$50 \%$ \\
flowering \\
\end{tabular} & $0.2548 * *$ & 1.0000 & $0.6575 * *$ & -0.1545 & $-0.2729 * *$ & -0.1234 & 0.1771 & -0.0783 & $\begin{array}{c}- \\
0.2313^{* *}\end{array}$ & 0.1506 & $-0.311 * * 1$ & 0.0319 & 0.0184 & -0.3530 \\
\hline \begin{tabular}{|c|} 
Number of \\
monopodia \\
per plant \\
\end{tabular} & $0.1803^{*}$ & $0.6086 * *$ & 000 & 618 & $-0.2571 * *$ & 0.0389 & $0.2803^{* *}$ & -0.1735 & $\begin{array}{c}- \\
0.3999 * *\end{array}$ & 0.2311 & -0.3377 & 0.2545 & 0.0853 & $-0.2304 * *$ \\
\hline \begin{tabular}{|c|} 
Number of \\
sympodia \\
per plant \\
\end{tabular} & $0.5621 * *$ & 05 & $-0.2284^{*}$ & 0 & $1 * *$ & 0.1156 & 0.0964 & $-0.2029^{*}$ & $\left|\begin{array}{c}- \\
0.298 * * 3\end{array}\right|$ & -0.0707 & -0. & 0.0525 & -0.0983 & $0.6196 * *$ \\
\hline \begin{tabular}{|c|}
$\begin{array}{c}\text { Number of } \\
\text { bolls per } \\
\text { plant }\end{array}$ \\
\end{tabular} & $0.1874 *$ & -0.1055 & -0.1245 & $0.4359 * *$ & 1.0000 & $0.4125^{* *}$ & $-0.1927 *$ & $0.2685 * *$ & -0.0944 & -0.0577 & 0.1086 & $0.3764 * *$ & $0.3596 * *$ & $0.9021 * *$ \\
\hline \begin{tabular}{|c|} 
Boll weight \\
$(\mathrm{g})$
\end{tabular} & 0.0901 & -0.1206 & 0.0004 & 0.1234 & -0.1213 & 1.0000 & $0.5293^{* *}$ & $0.2294 *$ & -0.1828 & $0.4135^{* *}$ & -0.1655 & -0.0290 & $0.2033^{*}$ & $0.2402 * *$ \\
\hline Seed index & 0.1688 & 0.1399 & $0.1954 *$ & 0.0099 & -0.1044 & $0.3943 * *$ & 1.0000 & $0.4953 * *$ & $* \begin{array}{c}- \\
0.2998 * *\end{array}$ & $0.4054 * *$ & -0.1515 & -0.0919 & $0.4169 * *$ & -0.0291 \\
\hline \begin{tabular}{|l|} 
Lint $\mathrm{i}$ \\
\end{tabular} & \begin{tabular}{|l|}
-0.0492 \\
\end{tabular} & 6669 & 1153 & 1662 & 0238 & 0.1523 & $0.5101^{* * *}$ & 1.0000 & $0.6765^{* *}$ & 0.1625 & -0.0863 & -0.0229 & $0.3344 * *$ & -0.2173 \\
\hline $\begin{array}{c}\text { Ginning out } \\
\text { turn }(\%)\end{array}$ & -0.2057 & -0.1891 & $-0.2820 * *$ & $-0.1945^{*}$ & 0.0394 & -0.1478 & $\begin{array}{c}- \\
0.2403 * * \\
\end{array}$ & $0.7065^{* *}$ & 1.0000 & $-0.1900 *$ & 0.0314 & 0.0618 & -0.0112 & $-0.2195^{*}$ \\
\hline $\begin{array}{c}2.5 \% \text { span } \\
\text { length }(\mathrm{mm})\end{array}$ & 0.0561 & 0.1267 & $0.1975^{*}$ & -0.1003 & -0.1456 & $0.2996 * *$ & $0.3119^{* *}$ & 0.1365 & -0.1235 & 1.0000 & $-0.8345^{* *}$ & -0.0937 & 0.8316 & -0.0235 \\
\hline \begin{tabular}{|c|}
$\begin{array}{c}\text { Uniformity } \\
\text { ratio }\end{array}$ \\
\end{tabular} & $\mid-0.0660$ & 1363 & 1040 & 2104 & $-0.2331 *$ & -0.1421 & -0.1387 & -0.0461 & 0.0650 & -0.1445 & 1.0000 & $-0.8979 * *$ & \begin{tabular}{|c|}
- \\
$0.7132 * *$ \\
\end{tabular} & $-0.3997 * *$ \\
\hline $\begin{array}{c}\text { Micronaire } \\
\text { value } \\
\left(10^{-6} \mathrm{~g} / \text { inch }\right)\end{array}$ & \begin{tabular}{|l|}
0.1234 \\
\end{tabular} & 0.0282 & $2062 *$ & 0.025 & -0.0792 & $\mid-0.0699$ & -0.1102 & -0.0735 & 0.0235 & 0.1593 & 0.0157 & 1.0000 & $|-\overline{-}|$ & 0.0049 \\
\hline $\begin{array}{l}\text { Bundle } \\
\text { strength } \\
\text { (g/tex) }\end{array}$ & \begin{tabular}{|l|}
-0.0189 \\
\end{tabular} & 0.0077 & 1189 & -0.1404 & 0.0158 & 0.1198 & $0.2517 * *$ & $0.2130^{*}$ & 0.0202 & $0.7414 * *$ & 0.0885 & 0.1214 & 1.0000 & 0.0418 \\
\hline \begin{tabular}{|c|} 
Seed cotton \\
yield per \\
plant \\
\end{tabular} & 0.2923 & -0.2847 & -0.1579 & 0.3897 & 0.4453 & 0.1658 & -0.0559 & -0.0960 & -0.0780 & 0.0542 & -0.0531 & 0.1338 & 0.1432 & 1.000 \\
\hline
\end{tabular}

* Significant at $5 \%$ level ** Significant at $1 \%$ level

Table 2: Direct and indirect effects (phenotypic) of 14 characters on seed cotton yield among 40 genotypes of cotton (Gossypium hirsutum L.) during kharif, 2019-20

\begin{tabular}{|c|c|c|c|c|c|c|c|c|c|c|c|c|c|}
\hline Character & \begin{tabular}{|c|} 
Plant \\
height
\end{tabular} & $\begin{array}{c}\text { Days to } \\
50 \% \\
\text { flowering } \\
\end{array}$ & $\begin{array}{c}\begin{array}{c}\text { Number of } \\
\text { monopodia per } \\
\text { plant }\end{array} \\
\end{array}$ & $\begin{array}{c}\text { Number of } \\
\text { sympodia } \\
\text { per plant }\end{array}$ & \begin{tabular}{|c|} 
Number of \\
bolls per \\
plant
\end{tabular} & $\begin{array}{c}\text { Boll } \\
\text { weight }\end{array}$ & $\begin{array}{c}\text { Seed } \\
\text { index }\end{array}$ & $\begin{array}{c}\text { Lint } \\
\text { index }\end{array}$ & $\begin{array}{l}\text { Ginning } \\
\text { outturn }\end{array}$ & \begin{tabular}{|l|}
$2.5 \%$ \\
span- \\
length \\
\end{tabular} & $\underset{\text { ratio }}{\text { Uniformity }}$ & $\begin{array}{c}\text { Micronaire } \\
\text { value }\end{array}$ & $\begin{array}{c}\text { Bundle } \\
\text { strength }\end{array}$ \\
\hline Plant height $(\mathrm{cm})$ & 0.2386 & 0.0608 & 0.0430 & 0.1341 & 0.0447 & 0.0215 & 0.0403 & -0.0117 & \begin{tabular}{|l|}
-0.0491 \\
\end{tabular} & 0.0134 & -0.0158 & 0.0294 & -0.0045 \\
\hline $\begin{array}{l}\text { Days to 50\% } \\
\text { flowering }\end{array}$ & $|-0.0661|$ & -0.2595 & -0.1579 & 0.0313 & 0.0274 & 0.0313 & -0.0363 & 0.0174 & 0.0491 & -0.0329 & 0.0354 & -0.0073 & -0.0020 \\
\hline $\begin{array}{c}\text { Number of } \\
\text { monopodia per } \\
\text { plant }\end{array}$ & -0.0050 & -0.0169 & -0.0278 & 0.0063 & 0.0035 & 0.0000 & -0.0054 & 0.0032 & 0.0078 & $-0.0055 \mid$ & 0.0029 & -0.0057 & -0.0033 \\
\hline \begin{tabular}{|c|} 
Number of \\
sympodia per plant
\end{tabular} & 0.0251 & -0.0054 & -0.0102 & 0.0447 & 0.0195 & 0.0055 & 0.0004 & -0.0074 & -0.0087 & $|-0.0045|$ & -0.0094 & -0.0011 & -0.0063 \\
\hline $\begin{array}{c}\text { Number of bolls per } \\
\text { plant }\end{array}$ & 0.0665 & -0.0374 & -0.0442 & 0.1546 & 0.3547 & -0.0 & -0.0 & -0.00 & 0 & $|-0.0516|$ & -0.0 & $-0 .($ & 0.0056 \\
\hline Boll weight (g) & 0.0177 & -0.0237 & 0.0001 & 0.0243 & -0.0239 & 0.1967 & 0.0775 & 0.0300 & -0.0291 & 0.0589 & -0.0279 & -0.0137 & 0.0236 \\
\hline Seed index & -0.0862 & -0.0714 & -0.0998 & 0.0051 & 0.0533 & -0.2014 & -0.5107 & -0.2605 & 0.1227 & -0.1593 & 0.0709 & 0.0563 & -0.1285 \\
\hline Lint index & -0.0259 & -0.0352 & -0.0606 & -0.0874 & -0.0125 & 0.0801 & 0.2681 & 0.5257 & 0.3714 & 0.0718 & -0.0242 & -0.0386 & 0.1120 \\
\hline $\begin{array}{c}\text { Ginning out turn } \\
(\%)\end{array}$ & 0.1178 & 0.1084 & 0.1616 & 0.1114 & -0.0226 & 0.0847 & 0.1377 & -0.4047 & -0.5729 & 0.0707 & -0.0373 & -0.0135 & -0.0116 \\
\hline $\begin{array}{l}2.5 \% \text { span length } \\
(\mathrm{mm})\end{array}$ & $|-0.0041|$ & -0.0093 & -0.0145 & 0.0074 & 0.0107 & -0.0220 & -0.0229 & -0.0100 & 0.0091 & $|-0.0736|$ & 0.0106 & -0.0117 & -0.0546 \\
\hline Uniformity ratio & -0.0003 & -0.0007 & -0.0005 & -0.0010 & -0.0011 & -0.0007 & -0.0007 & -0.0002 & 0.0003 & -0.0007 & 0.0049 & 0.0001 & 0.0004 \\
\hline $\begin{array}{c}\text { Micronaire value } \\
\left(10^{-6} \mathrm{~g} / \text { inch }\right)\end{array}$ & 0.0178 & 0.0041 & 0.0297 & -0.0037 & -0.0114 & $|-0.0101|$ & -0.0159 & 106 & 0034 & 0.0230 & .0023 & .1442 & 0.0175 \\
\hline $\begin{array}{c}\text { Bundle } \\
\text { strength }(\mathrm{g} / \mathrm{tex})\end{array}$ & -0.0037 & 0.0015 & 0.0232 & -0.0274 & 0.0031 & 0.0233 & $0.0490 \mid$ & 0.0415 & 0.0039 & 0.1445 & 0.0172 & 0.0237 & 0.1948 \\
\hline $\begin{array}{c}\text { Correlation with } \\
\text { seed cotton yield } \\
\text { per plant }\end{array}$ & 0.2923 & -0.2847 & -0.1579 & 0.3897 & 0.4453 & 0.1658 & -0.0559 & -0.0960 & -0.0780 & 0.0542 & -0.0531 & 0.1338 & 0.1432 \\
\hline
\end{tabular}


/plant (g)

* Significant at 5\% level, ** Significant at $1 \%$ level, Residual effect $=0.539$, Bold and diagonal values indicate direct effects

Table 3: Direct and indirect effects (genotypic) of 14 characters on seed cotton yield among 40 genotypes of cotton (Gossypium hirsutum L.) during kharif, 2019-20

\begin{tabular}{|c|c|c|c|c|c|c|c|c|c|c|c|c|c|}
\hline Character & $\begin{array}{c}\text { Plant } \\
\text { height }\end{array}$ & $\begin{array}{c}\text { Days to } \\
50 \% \\
\text { flowering }\end{array}$ & $\begin{array}{l}\text { Number of } \\
\text { monopodia } \\
\text { per plant }\end{array}$ & $\begin{array}{c}\text { Number } \\
\text { of } \\
\text { sympodia } \\
\text { per plant }\end{array}$ & $\begin{array}{c}\text { Number } \\
\text { of bolls } \\
\text { per plant }\end{array}$ & $\begin{array}{c}\text { Boll } \\
\text { weight }\end{array}$ & $\begin{array}{c}\text { Seed } \\
\text { index }\end{array}$ & $\begin{array}{c}\text { Lint } \\
\text { index }\end{array}$ & $\begin{array}{l}\text { Ginning } \\
\text { outturn }\end{array}$ & $\begin{array}{c}2.5 \% \\
\text { span } \\
\text { length }\end{array}$ & $\begin{array}{c}\text { Uniformity } \\
\text { ratio }\end{array}$ & $\begin{array}{c}\text { Micronaire } \\
\text { value }\end{array}$ & $\begin{array}{c}\text { Bundle } \\
\text { strength }\end{array}$ \\
\hline $\begin{array}{l}\text { Plant height } \\
(\mathrm{cm})\end{array}$ & 1.4106 & 0.4604 & 0.3161 & 1.1380 & 0.5015 & 0.0878 & 0.4979 & $\left|\begin{array}{c}- \\
0.1611\end{array}\right|$ & -0.6026 & $\left|\begin{array}{c}- \\
0.0975\end{array}\right|$ & -0.5445 & 0.0302 & -0.2507 \\
\hline $\begin{array}{c}\text { Days to } 50 \% \\
\text { flowering }\end{array}$ & $\begin{array}{c}- \\
0.1181\end{array}$ & -0.3619 & -0.2380 & 0.0559 & 0.0988 & 0.0446 & $\begin{array}{c}- \\
0.0641 \\
\end{array}$ & 0.0283 & 0.0837 & $\begin{array}{c}- \\
0.0545\end{array}$ & 0.1126 & -0.0116 & -0.0067 \\
\hline $\begin{array}{l}\text { Number of } \\
\text { monopodia } \\
\text { per plant }\end{array}$ & $\begin{array}{c}- \\
0.1151\end{array}$ & -0.3376 & -0.5135 & 0.1345 & 0.1320 & $\begin{array}{c}- \\
0.0200\end{array}$ & $\begin{array}{c}- \\
0.1439\end{array}$ & 0.0891 & 0.2053 & $\mid \begin{array}{c}- \\
0.1187\end{array}$ & 0.1734 & -0.1307 & -0.0438 \\
\hline $\begin{array}{c}\text { Number of } \\
\text { sympodia per } \\
\text { plant }\end{array}$ & $\begin{array}{c}- \\
0.7966\end{array}$ & 0.1525 & 0.2586 & -0.9874 & -0.5017 & $\begin{array}{c}- \\
0.1141\end{array}$ & $\begin{array}{c}- \\
0.0952\end{array}$ & 0.2003 & 0.2945 & 0.0698 & 0.2868 & -0.0519 & 0.0971 \\
\hline \begin{tabular}{|c|} 
Number of \\
bolls per plant
\end{tabular} & 0.0882 & -0.0677 & -0.0638 & 0.1260 & 0.2480 & $\begin{array}{c}- \\
0.1023 \\
\end{array}$ & $\begin{array}{c} \\
0.0478 \\
\end{array}$ & $\begin{array}{c}- \\
0.0666\end{array}$ & -0.0234 & $\begin{array}{c}- \\
0.0143 \\
\end{array}$ & 0.0269 & 0.0934 & 0.0892 \\
\hline $\begin{array}{c}\text { Boll weight } \\
(\mathrm{g})\end{array}$ & 0.0501 & -0.0994 & 0.0313 & 0.0931 & -0.3322 & 0.8055 & 0.4264 & 0.1848 & -0.1473 & 0.3331 & -0.1333 & -0.0234 & 0.1637 \\
\hline Seed index & 0.0050 & 0.0025 & 0.0039 & 0.0014 & -0.0027 & 0.0074 & 0.0140 & 0.0070 & -0.0042 & 0.0057 & -0.0021 & -0.0013 & 0.0059 \\
\hline Lint index & 0.1268 & 0.0870 & 0.1928 & 0.2254 & 0.2983 & $\begin{array}{c}- \\
0.2549 \\
\end{array}$ & $\begin{array}{c}- \\
0.5503 \\
\end{array}$ & $\begin{array}{c}- \\
1.1109\end{array}$ & -0.7516 & $\begin{array}{c}- \\
0.1805\end{array}$ & 0.0959 & 0.0254 & -0.3715 \\
\hline $\begin{array}{c}\text { Ginning out } \\
\text { turn }(\%)\end{array}$ & $\begin{array}{c}- \\
0.2436 \\
\end{array}$ & -0.1319 & -0.2280 & -0.1701 & -0.0539 & $\begin{array}{c}- \\
0.1043 \\
\end{array}$ & $\begin{array}{c}- \\
0.1710 \\
\end{array}$ & 0.3858 & 0.5703 & $\begin{array}{c}- \\
0.1083 \\
\end{array}$ & 0.0179 & 0.0352 & -0.0064 \\
\hline $\begin{array}{c}2.5 \% \text { span } \\
\text { length }(\mathrm{mm})\end{array}$ & 0.0564 & -0.1229 & -0.1886 & 0.0577 & 0.0471 & $\begin{array}{c}- \\
0.3374\end{array}$ & $\begin{array}{c}- \\
0.3308 \\
\end{array}$ & $\begin{array}{c}- \\
0.1326\end{array}$ & 0.1550 & $\begin{array}{c}- \\
0.8161\end{array}$ & 0.6810 & 0.0765 & -0.6787 \\
\hline $\begin{array}{l}\text { Uniformity } \\
\text { ratio }\end{array}$ & 0.0472 & 0.0380 & 0.0413 & 0.0355 & -0.0133 & 0.0202 & 0.0185 & 0.0106 & -0.0038 & 0.1020 & -0.1222 & 0.1097 & 0.0872 \\
\hline $\begin{array}{c}\text { Micronaire } \\
\text { value } \\
\left(10^{-6} \mathrm{~g} / \mathrm{inch}\right) \\
\end{array}$ & 0.0057 & 0.0084 & 0.0672 & 0.0139 & 0.0994 & $\begin{array}{c}- \\
0.0077\end{array}$ & $\begin{array}{c}- \\
0.0243\end{array}$ & $\begin{array}{c}- \\
0.0060\end{array}$ & 0.0163 & $\mid \begin{array}{c}- \\
0.0247\end{array}$ & -0.2370 & 0.2639 & -0.1024 \\
\hline $\begin{array}{l}\text { Bundle } \\
\text { strength } \\
\text { (g/tex) }\end{array}$ & $\begin{array}{c}- \\
0.1882\end{array}$ & 0.0195 & 0.0903 & -0.1041 & 0.3808 & 0.2153 & 0.4415 & 0.3541 & -0.0118 & 0.8806 & -0.7552 & -0.4108 & 1.0589 \\
\hline $\begin{array}{l}\text { Correlation } \\
\text { with seed } \\
\text { cotton yield } \\
\text { per plant } \\
\text { /plant }(\mathrm{g})\end{array}$ & 0.3284 & -0.3530 & -0.2304 & 0.6196 & 0.9021 & 0.2402 & $\begin{array}{c}- \\
0.0291\end{array}$ & $\mid \begin{array}{c}- \\
0.2173\end{array}$ & -0.2195 & $\mid \begin{array}{c}- \\
0.0235\end{array}$ & -0.3997 & 0.0049 & 0.0418 \\
\hline
\end{tabular}

$*=$ Significant at 5\% level, $* *=$ Significant at $1 \%$ level, Residual effect $=0.1245$, Bold and diagonal values indicate direct effects

\section{Conclusion}

The results discussed above indicate that correlation and direct and indirect effect estimates vary for different traits with variation in genetic material based on yield component traits and fibre properties. Hence, correlations and direct and indirect effect estimation would provide useful information for planning a successful breeding programme if the genetic material is grouped for yield and fibre quality characters and also it is essential to device suitable breeding methodologies for simultaneous improvement of both yield and quality parameters involving three way crosses, modified back crosses or recurrent selection.

\section{Reference}

1. Ahsan Muhammad Zahir, Muhammad Saffar Majidano, Hadayatullah Bhutto, Abdul Razzaque Channa, Karim Bakhsh Sial, Allah Dino Kalhoro. Genetic variability and correlation analysis of some upland cotton (Gossypium hirsutum L.) accessions. International Journal of Scientific \& Engineering Research. 2014; 5:3.

2. Al-Jibouri HA, Millar PA, Robinson HP. Genotypic and environmental variances and covariances is an upland cotton cross of interspecific origin. Agron. J. 1958; 50:633-636.

3. Alkuddsi YA, Gururaja Rao MR, Patil SS, Gowda TH, Joshi M. Combining ability analysis for seed cotton yield (Kapas Yield) and its components in intra hirsutum hybrids and forming heterotic boxes for exploitation in cotton. Genomics Applied Biology. 2013; 4(5):35-49.

4. An DTH, Ravikesavan R, Iyanar K. Genetic advance and heritability as a selection index for improvement of yield and quality in cotton. Journal of Cotton Research and Development. 2008; 22(1):14-18.

5. Asha R, Ahmed ML, Babu DR, Kumar PA. Character association and path coefficient analysis for yield and component traits in upland cotton. Journal of Cotton Research and Development. 2015; 29(1):31-35.

6. Ashokkumar, Ravikesavan R. Genetic studies of correlation and path coefficient analysis for seed oil, yield and fibre quality traits in cotton (G. hirsutum L.) Aust. J Basic. Appl. Sci. 2010; 4(11):5496-5499.

7. Desalegn Z, Ratanadilok N, Kaveeta R. Correlation and heritability for yield and fiber quality parameters of Ethiopian Cotton (Gossypium hirsutum L.) estimated 
from 15 (diallel) crosses. Kasetsart J (Nat. Sci.). 2009; 43:1-11.

8. Dewey DR, Lu RH. A correlation and path coefficient analysis of components of crested wheatgrass seed production. Agron. J. 1959; 51:515-518.

9. Erande CS, Kalpande HV, Deosarkar DB, Chavan SK, Patil VS, Deshmukh JD et al. Genetic variability, correlation and path analysis among different traits in desi cotton (Gossypium arboretum L.). African Journal of Agricultural Research. 2014; 9(29):2278-2286.

10. Hazem Bayaty. Path coefficient analysis in upland cotton (Gossypium hirsutum L.). Mesopotamia J Agric. 2005; 33:3-9.

11. Iqbal M, Hayat K, Khan RSA, Sadiq A, Islam N. Correlation and path coefficient analysis of earliness and yield traits in cotton ( $G$. hirsutum L.) Asian J Pl. Sci. 2006; 5(2):341-344.

12. Kaushik SK, Singhania DL, Kapoor CJ. Correlation and path analysis among different traits in upland cotton (Gossypium hirsutum L). J cott. Res. and Dev. 2005; 19(2):140-144.

13. Kishore DK, Gopinath M, Rao VS, Rao VS. Genetic variability studies in upland cotton. The Andhra Agricultural Journal. 2011; 58(1):17-21.

14. Kumar KA, Ravikesavan R. Genetic Studies of correlation and path coefficient analysis for seed oil, yield and fibre quality traits in cotton (Gossypium hirsutum L.). Australian Journal of Basic and Applied Sciences. 2010; 31(5):861-864.

15. Panse VG, Sukhatme PV. Statistical Methods for Agricultural Workers: Indian Council of Agricultural Research, New Delhi, 1978.

16. Preetha S, Raveendran TS. Genetic variability and association analysis in three different morphological groups of cotton (Gossypium hirsutum L.) Asian J Pl. Sci. 2007; 6(1):122-128.

17. Proceedings of Annual group meetings of AICRIP on cotton, 2018-19.

18. Pujer S, Siwach SS, Deshmukh J, Sangwan RS, Sangwan O. Genetic variability, correlation and path analysis in upland cotton (Gossypium hirsutum L.). Electronic Journal of Plant Breeding. 2014; 5(2):284-289.

19. Rajamani S, Sumalatha T, Gopinath M. Correlation and path analysis in upland cotton (Gossypium hirsutum. L.). Journal of Cotton Research and Development. 2013; 27(2):188-190.

20. Ramesh. genome mapping and identification of molecular markers for various agronomic and fibre quality traits in tetraploid cotton. Ph. D. Thesis, Univ. Agric. Sci, Dharwad, Karnataka (India), 2015.

21. Sirisha ABM, Lal Ahamed M, Rama Kumar PV, Ratnakumari S, Srinivasa Rao V. Correlation and path analysis studies in cotton (Gossypium hirsutum L.). The Andhra Agricultural Journal. 2016; 63(1):104-109.

22. Vinodhana KN, Gunasekaran M, Vindhiyavarman P. Genetic Studies of Variability, Correlation and Path Coefficient analysis in Cotton genotypes. Int. J Pure App. Biosci. 2013; 1(5):6-10. 